\title{
Does metformin have a "buffer effect" on serum TSH levels in euthyroid diabetic patients?
}

\author{
Silvia Santos-Palacios, ${ }^{1}$ Antonio Brugos-Larumbe, ${ }^{2}$ Francisco Guillén-Grima, ${ }^{3}$ \\ Ane Garmendia-Madariaga, ${ }^{4}$ Juan C. Galofré ${ }^{1}$
}

${ }^{1}$ Department of Endocrinology and Nutrition, Clínica Universidad de Navarra, University of Navarra, ${ }^{2}$ Department of Health Sciences, Public University of Navarra, ${ }^{3}$ Department of Preventive Medicine, Clínica Universidad de Navarra, University of Navarra, Pamplona, Spain, ${ }^{4}$ Department of General Surgery, Spital Zollikerberg, Zürich, Switzerland

\begin{abstract}
OBJECTIVE: In the last few years a number of studies have found that metformin has a thyrotropin (TSH)-lowering effect in patients with type 2 diabetes (DM2) and that this effect disappears after discontinuation of the drug. Initial studies were carried out in hypothyroid patients on levothyroxine (LT4) treatment. However, this effect was observed only when serum TSH levels were elevated. The aim of this study was to assess the effect of metformin treatment on serum TSH levels in euthyroid DM2 patients. DESIGN: A retrospective study was conducted in seven primary health care centers in Spain. The study included 278 DM2 patients (110 females). Serum TSH level was tested before and one year after the onset of metformin treatment. We compared both TSH levels by paired $t$-tests. The sample was divided into four hierarchical clusters (C1 to C4), according to the Euclidean distance of the initial TSH level. No patient received LT4 treatment. RESULTS: Pre-metformin serum TSH level $(2.00 \pm 0.76 \mathrm{mU} / \mathrm{L})$ was statistically significantly lower than post-metformin level $(2.20 \pm 0.87 \mathrm{mU} / \mathrm{L} ; P<0.001)$. Interestingly, this trend was observed only in clusters 1 and 2 (those with lower baseline TSH levels), whereas the trend was lost in clusters 3 and 4 . The difference between pre- and post-metformin TSH levels follows a mathematical model. According to this model, the TSH threshold point level is $3.00 \mathrm{mU} / \mathrm{L}$. CONCLUSION: Metformin seems to induce a "buffer effect" on TSH secretion in euthyroid diabetic individuals. Hence, our study found an unreported TSH behavior after metformin treatment.
\end{abstract}

Key words: Metformin, Thyroid, Thyrotropin, Type 2 diabetes

Address for correspondence:

Dr. Juan C. Galofré, Department of Endocrinology and Nutrition, Clínica Universidad de Navarra,

Pio XII, 36, 31080 Pamplona, Spain,

Tel.: 948255 400. Fax: 948296 500,

E-mail: jcgalofre@unav.es

Received: 10-07-2014, Accepted:14-10-2014

\section{INTRODUCTION}

Type 2 diabetes mellitus (DM2) and thyroid dysfunction are the two most prevalent endocrine disorders. ${ }^{1,2}$ In addition, the prevalence of thyroid dysfunction in DM2 patients is higher than in the general population..$^{3-5}$ 
Metformin is considered the first choice as an oral agent for individuals with $\mathrm{DM} 2^{6}$ and may also be prescribed when insulin resistance is present in women with polycystic ovarian syndrome (PCOS), ${ }^{7,8}$ The TSH-lowering effect of metformin in DM2 patients with primary hypothyroidism was first reported by Vigersky et al in 2006. ${ }^{9}$ This effect was observed in both hypothyroid patients under levothyroxine (LT4) replacement therapy and in untreated subjects. Since then, several retrospective and prospective studies have confirmed this observation. ${ }^{10,11}$ While the majority of the initial studies were carried out on hypothyroid patients, other investigators recruited euthyroid subjects yielding, however, perplexing results. ${ }^{11-13}$ Furthermore, the aforementioned effect of metformin was also observed in hypothyroid women with coexistent PCOS. ${ }^{14,15}$

Although the potential influence of LT4 therapy on the TSH-lowering effect of metformin in subjects under metformin treatment was ruled out in the previous studies, its presence could be misleading. Therefore, we decided to analyze a sample of patients not taking any thyroid hormone replacement. The available information led us to hypothesize that, to some extent, the metformin effect on TSH secretion could depend on the hormone circulating concentration. ${ }^{16}$ Hence, our study was designed to clarify this issue. We decided to evaluate the variation in serum TSH levels after one year of metformin treatment in a sample of DM2 patients with normal thyroid function. In our sample no patients were undergoing LT4 treatment.

\section{SUBJECTS AND METHODS}

We conducted a retrospective study in Navarra (northern Spain). DM2 patients on metformin treatment were recruited through the regional registry computerized database from seven primary health care centers. In these centers all the blood test results have been electronically compiled since 2004 . Screening of thyroid function by evaluation of serum TSH level has been routinely performed on all individuals seeking medical advice in our region. Serum-free thyroxin (FT4) and thyroid autoantibodies levels are not routinely measured as part of the screening program, although they could be ordered at the discretion of the attending physician.
After obtaining the institutional approval of the Ethical Committee of the Public University of Navarra, we reviewed the electronic records of the selected Health Care Centers. We identified 4,007 DM2 patients taking metformin. Of the 4,007 potentially eligible patients, 406 patients had had a TSH determination before and after the onset of metformin treatment with one year apart in their follow-up. We rejected patients on LT4 treatment. Eventually we identified 278 euthyroid subjects (TSH within the reference range of $0.72-4.43 \mathrm{mU} / \mathrm{mL}$ ) who satisfied the inclusion criteria for the study (Figure 1). The study was classified by the Spanish Medicine Agency (AEMPS) as an EPA-OD (Estudio Postautorizacion con Otros Diseños diferentes al de seguimiento prospectivo) with the code number ABL-MET-2013-01 on December 9, 2013.

The sample was further divided into hierarchical clusters $(\mathrm{C} 1$ to $\mathrm{C} 4)$ for analysis according to the $\mathrm{Eu}-$ clidean distance of the initial (pre-metformin) TSH level. A dendrogram was performed to decide the number of clusters to be used.

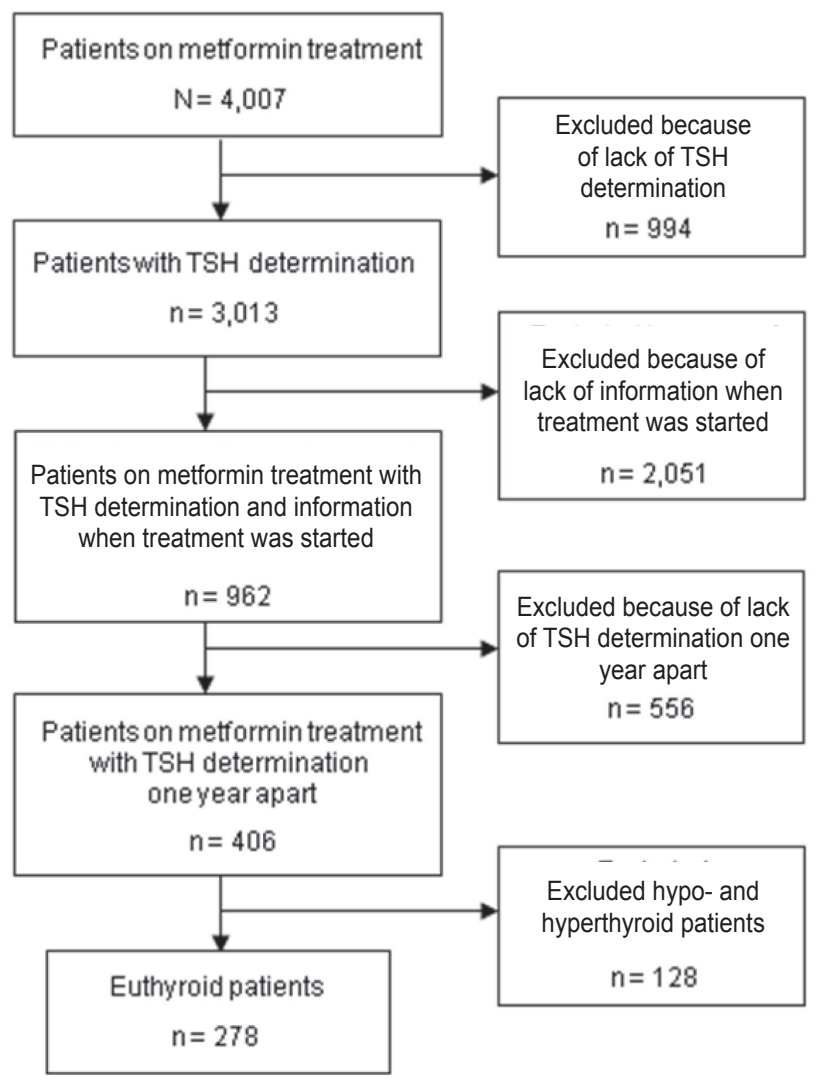

Figure 1. Flow chart of the study patients. 
Information regarding height, weight and blood pressure was also included in the study. Body mass index (BMI) was calculated as body weight (in $\mathrm{kg}$ ) divided by the square of body height (in meters). Venous blood samples were drawn after 12 hours fasting in a baseline condition.

\section{Laboratory measurements}

Serum concentration of TSH was analyzed using a chemiluminescent immunoassay TSH3-Ultra (ADVIA Centaur ${ }^{\circledast}$ CP Immunoassay System). Inter-assay coefficient of variation is below $6 \%$. TSH reference limits ranged between 0.72 and $4.43 \mathrm{mU} / \mathrm{L}$, according to our findings in a previous study. ${ }^{17}$

\section{Statistical analyses}

Statistical analyses were performed using SPSS for Windows (version 17.0; SPSS, Inc., Chicago, IL, USA). Normal distributions were assessed with the Kolmogorov-Smirnov test, as well as skewness and kurtosis. The descriptive analysis was performed using mean and standard deviations for continuous normal variables or median values and $25^{\text {th }}-75^{\text {th }}$ percentiles for heavily skewed variables. Paired $t$-tests were used to analyze changes in TSH levels after metformin treatment. Groups were defined using cluster analysis. Linear regression analysis was performed to assess metformin effect on serum TSH level. $P$ values were considered statistically significant when $P<0.05$.

\section{RESULTS}

A total of 278 euthyroid diabetic individuals ful- filled the inclusion criteria. The sample was divided into 4 clusters. The demographics and serum TSH levels (at baseline and post-metformin therapy) of the sample and clusters are displayed in Table 1.

Studied as a whole, and in contrast to what was expected, post-metformin serum TSH level was statistically significantly higher than the pre-metformin level $(2.20 \pm 0.87$ vs. $2.00 \pm 0.76 \mathrm{mU} / \mathrm{L} ; P<0.001)$. However, the analysis by clusters revealed that this trend was observed only in clusters 1 and 2 (those with lower basal TSH levels), whereas this trend was lost in clusters 3 and 4 (those with the lowest number of patients: 32 and 13 patients, respectively) (Table 1 and Figure 2).

The analysis of the changes in TSH level depicted similar results in both genders. In males they varied from $1.93 \pm 0.74$ to $2.13 \pm 0.85 \mathrm{mU} / \mathrm{L}, P<0.001$, and in females from $2.11 \pm 0.79$ to $2.31 \pm 0.90 \mathrm{mU} / \mathrm{L}, P$ $<0.001$ (Figure 3).

A linear regression model showed an inverse correlation between the basal and post-metformin serum TSH levels. The lower the initial TSH, the higher the TSH will be after metformin treatment (Figure 4). The difference between post-metformin and pre-metformin serum TSH level is explained by the following mathematical model: Difference $($ Post-Previous $)=0.607-0.204$ (baseline TSH) $P$ $<0.001$. The variation from basal to post-metformin serum TSH level was more than $40 \%$ in $19.8 \%$ of euthyroid subjects.

Table 1. Clinical and biochemical data of euthyroid diabetic patients

\begin{tabular}{lccccc}
\hline & \multicolumn{5}{c}{ Euthyroid Diabetic Patients } \\
\cline { 2 - 6 } & All & Cluster 1 & Cluster 2 & Cluster 3 & Cluster 4 \\
\hline Patients $(\mathrm{N})$ & 278 & 95 & 138 & 32 & 13 \\
Gender M/F [N (\%)] & $168(60.4) / 110(39.6)$ & $64(67.4) / 31(32.6)$ & $83(60.1) / 55(39.9)$ & $15(46.9) / 17(53.1)$ & $6(46.1) / 7(53.9)$ \\
Age (years) & $70.23 \pm 11.39$ & $71.87 \pm 11.74$ & $69.92 \pm 11.28$ & $67.57 \pm 10.70$ & $68.25 \pm 11.13$ \\
BMI & $30.20 \pm 5.0$ & $29.33 \pm 4.82$ & $30.36 \pm 4.83$ & $31.47 \pm 5.30$ & $31.54 \pm 6.68$ \\
Pre-metformin TSH & $2.00 \pm 0.76$ & $1.20 \pm 0.60$ & $2.14 \pm 0.34$ & $3.08 \pm 0.16$ & $3.75 \pm 0.19$ \\
Post-metformin TSH & $2.20 \pm 0.87^{*}$ & $1.53 \pm 0.22^{*}$ & $2.33 \pm 0.69^{*}$ & $3.02 \pm 0.66^{*}$ & $3.66 \pm 0.63^{\#}$ \\
\hline
\end{tabular}

Data are expressed as mean \pm SD or as a percentage $(\%)$. N, number of patients. M, males. F, females. BMI $\left(\mathrm{kg} / \mathrm{m}^{2}\right)$, body mass index. TSH (mU/L) reference range: 0.72-4.43. Difference between pre-metformin and post-metformin serum TSH levels: ${ }^{*} P<0.01$; ${ }^{\&} P=0.19 ;{ }^{*} P=0.33$. 


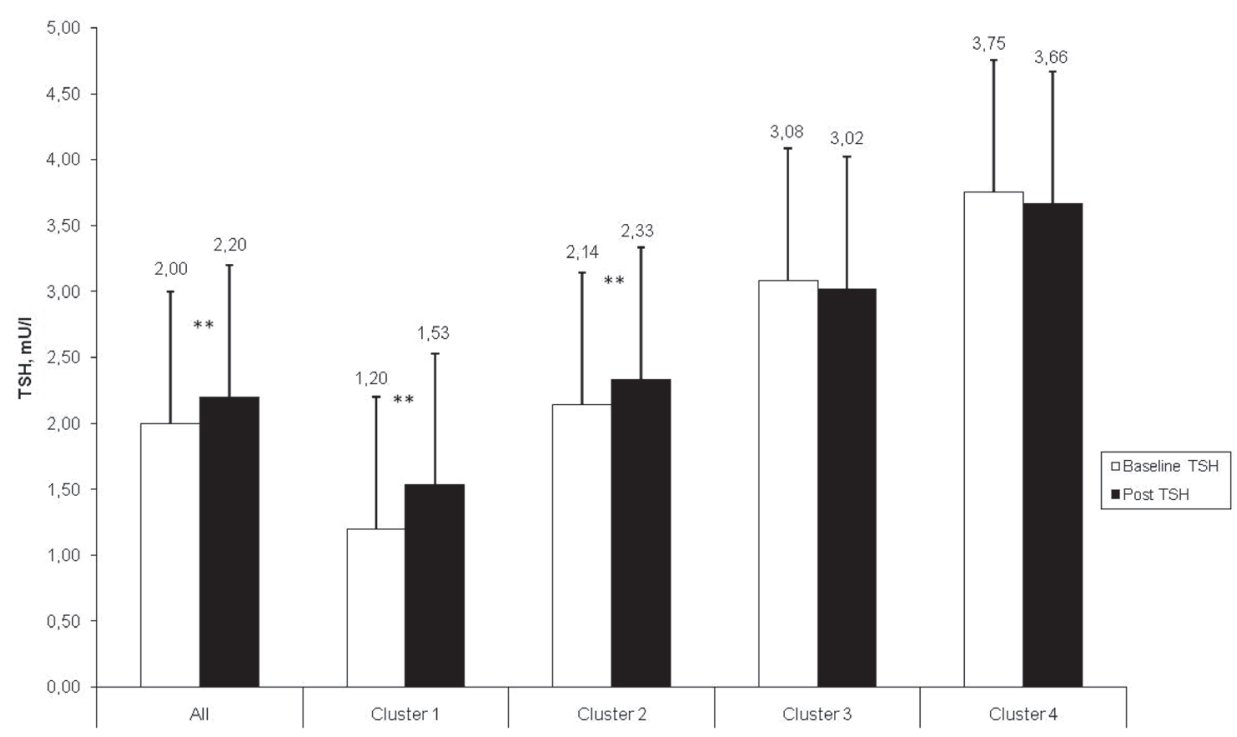

Figure 2. The effect of metformin on serum TSH in euthyroid diabetic patients (subdivided into clusters). ${ }^{* *} P<0.01 ;{ }^{*} P<0.05$.

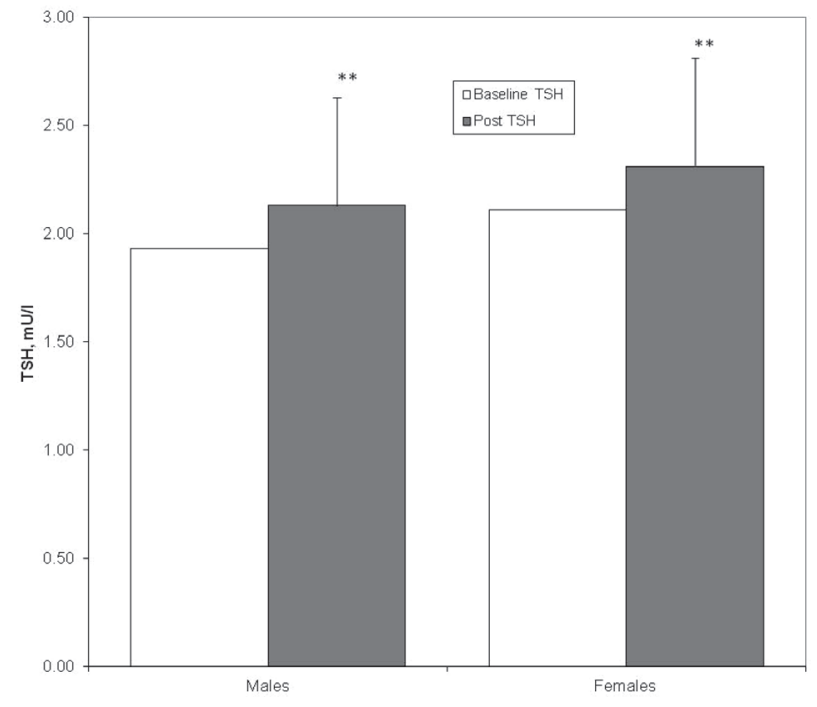

Figure 3. The effect of metformin on serum TSH in males and females. ${ }^{* *} P<0.01$.

\section{DISCUSSION}

The results of the present retrospective study suggest that metformin treatment shifts circulating TSH concentration away from both ends of the spectrum of the reference range in euthyroid DM2 patients. We found a slight but significant increase of TSH level when TSH was in the lower-normal range, whereas a slight, although insignificant decrease, was observed when it was in the upper-normal range. We note that

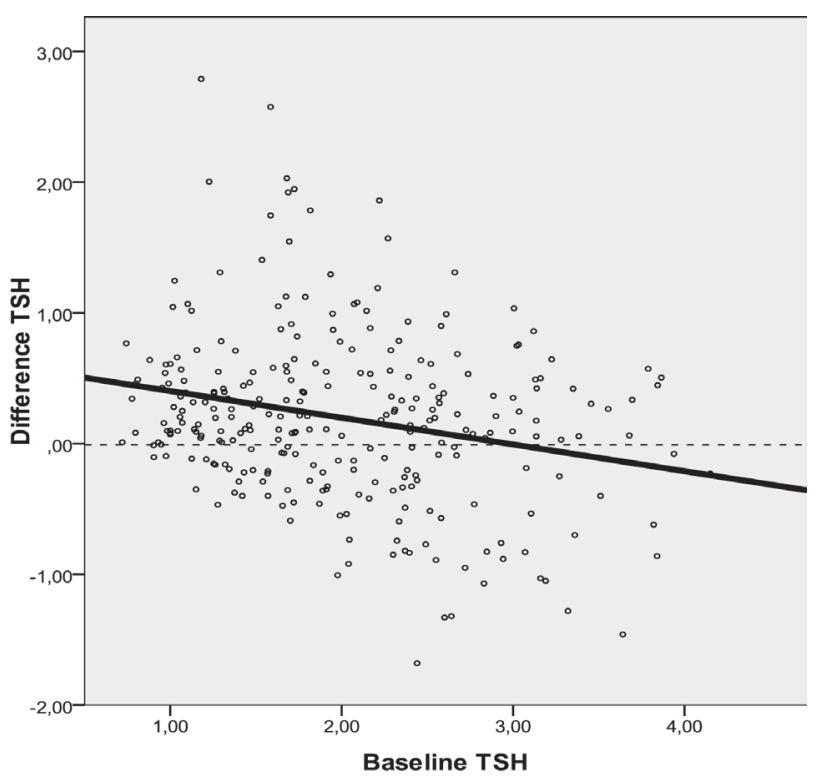

Figure 4. Correlation between baseline TSH (mU/l) and change in TSH (difference: post - previous TSH) after metformin treatment. Linear regression analysis. $\mathrm{R}=0.240 ; P<0.01$.

clusters 3 and 4 had the lowest number of patients (32 and 13, respectively) and this circumstance might affect the result and its significance. This unreported TSH behavior after metformin treatment is what we have dubbed the metformin "buffer effect", since it seems that metformin induces in circulating TSH a return to the middle of the normal range. 
Some previous studies have reported the absence of apparent changes in TSH levels after metformin treatment in euthyroid subjects. ${ }^{11,13,18-20}$ Our results are in contrast with these previous findings and we believe that the discrepancy could be explained by the difference in the study design. A prospective study by the Cappelli group examined 54 euthyroid patients with DM2. ${ }^{11}$ In this particular population metformin did not have a significant effect on serum TSH level, suggesting that metformin has a neutral effect on TSH in euthyroid individuals. Furthermore, a recent study of 828 DM2 patients with normal thyroid function by Díez \& Iglesias in Spain with a cross-sectional design did not bear out a relationship between TSH values and metformin treatment in euthyroid DM2 patients. ${ }^{13}$ Rezzonico et al included only women with insulin resistance and thyroid nodules. ${ }^{18}$ Oleandri et al selected a short sample of 28 subjects with abdominal obesity and their patients were treated with metformin for a short period of 3 months. ${ }^{19}$ The published meta-analysis ${ }^{20}$ found no change in TSH levels in euthyroid patients, but was based only on the 2009 Cappelli study. ${ }^{11}$

The results of our study are consistent with those of Cappelli et al published in 2012. ${ }^{12}$ In this second paper they designed a large retrospective study analyzing 393 DM2 patients. They corroborated the metformin-lowering TSH effect on subjects with highnormal basal serum TSH levels and on hypothyroid patients as well. These controversial findings clearly indicated the need for an in-depth analysis to explain the difference. Our suggested metformin "buffer effect" could help to explain the discrepancy, indicating that the investigators need to thoroughly dissect their data. It seems that not all "normal range" values are equally "normal".

The difference between pre- and post-metformin TSH levels follows a mathematical model. According to this model, the TSH cut-off point level is 2.98 $\mathrm{mU} / \mathrm{L}$. In other words, metformin will have a lowering effect on TSH in those individuals with a serum TSH level higher than $2.98 \mathrm{mU} / \mathrm{L}$, whereas this effect will be the opposite when basal TSH level is lower than $2.98 \mathrm{mU} / \mathrm{L}$. Therefore we assume that a cutoff of $3 \mathrm{mU} / \mathrm{L}$ can be established that separates the TSH trend of this metformin "buffer effect".
Our sample accurately represents the general population of our region. The fact that we included all individuals who seek medical advice from several general health care centers minimizes the potential selection bias of the study. In order to validate our results, we also analyzed the changes in circulating TSH level one year apart in a control group of euthyroid patients free of metformin treatment. We found no differences in circulating pre- and post- TSH levels. Since obesity could be considered as a possible confounding factor, we additionally analyzed serum TSH levels in obese and non-obese patients and observed the same trend in both groups (data not shown).

The mechanism of the metformin TSH-lowering effect remains largely unknown. ${ }^{20}$ However, it has been speculated that metformin may modulate thyroid function at central and tissue level. ${ }^{23}$ Previous observations have described how complex and multifactorial the metformin action on TRH/TSH/ T4 could be. ${ }^{9}$ Metformin may change the affinity of and/or the number of thyroid hormone receptors, increase the dopaminergic tone in the pituitary or induce a constituent activation of the TSH receptor., ${ }^{9,21}$ It has also been proposed that metformin may have an inhibitory effect on hypothalamic AMPK activity which counteracts $\mathrm{T} 3$ effects at this level..$^{22,23}$ In addition, metformin seems to improve peripheral thyroid function in both LT4-treated and LT4-untreated patients with hypothyroidism. ${ }^{20}$ The observation that the TSH-lowering effect of metformin also occurs in LT4-untreated hypothyroid patients excludes the possibility that the effect is due to an increased LT4 absorption in the gastrointestinal tract. ${ }^{20}$

Although metformin-induced TSH changes seem to be small, this reduction could have a relevant impact on individuals with untreated subclinical hypothyroidism. This effect could also be relevant in obese euthyroid DM2 subjects, who normally have slightly increased TSH levels, ${ }^{17,24}$ in whom the use of metformin is indicated as treatment for insulin resistance.

In conclusion, our findings offer an insight into the effect of metformin on thyroid homeostasis which seems to be modulated according to the individual set point of circulating TSH levels. These data indicate that the repercussion of metformin treatment on serum TSH level in euthyroid DM2 patients resembles 
a "buffer effect". This could be of especial clinical relevance in euthyroid patients with serum TSH level in the normal-upper limit.

\section{DECLARATION OF INTEREST}

The authors declare that there is no conflict of interest.

\section{FUNDING}

This research did not receive any specific grant from any funding agency in the public, commercial or not-for-profit sector.

\section{REFERENCES}

1. Wild S, Roglic G, Green A, Sicree R, King H, 2004 Global prevalence of diabetes: estimates for the year 2000 and projections for 2030. Diabetes Care 27: 1047-1053.

2. Canaris GJ, Manowitz NR, Mayor G, Ridgway EC, 2000 The Colorado thyroid disease prevalence study. Arch Intern Med 160: 526-534.

3. Perros P, McCrimmon RJ, Shaw G, Frier BM, 1995 Frequency of thyroid dysfunction in diabetic patients: value of annual screening. Diabet Med 12: 622-627.

4. Wu P, 2007 Thyroid disorders and diabetes. It is common for a person to be affected by both thyroid disease and diabetes. Diabetes Self Manag 24: 80-82, 85-87.

5. Chubb SA, Davis WA, Inman Z, Davis TM, 2005 Prevalence and progression of subclinical hypothyroidism in women with type 2 diabetes: the Fremantle Diabetes Study. Clin Endocrinol (Oxf) 62: 480-486.

6. Nathan DM, Buse JB, Davidson MB, et al, American Diabetes Association; European Association for the Study of Diabetes; 2009 Medical management of hyperglycemia in type 2 diabetes: a consensus algorithm for the initiation and adjustment of therapy: a consensus statement of the American Diabetes Association and the European Association for the Study of Diabetes. Diabetes Care 32: 193-203.

7. Velazquez EM, Mendoza S, Hamer T, Sosa F, Glueck CJ, 1994 Metformin therapy in polycystic ovary syndrome reduces hyperinsulinemia, insulin resistance, hyperandrogenemia, and systolic blood pressure, while facilitating normal menses and pregnancy. Metabolism 43: 647-654.

8. Dunaif A, 2008 Drug insight: insulin-sensitizing drugs in the treatment of polycystic ovary syndrome-a reappraisal. Nat Clin Pract Endocrinol Metab 4: 272-283.

9. Vigersky RA, Filmore-Nassar A, Glass AR, 2006 Thyrotropin suppression by metformin. J Clin Endocrinol Metab 91: 225-227.

10. Isidro ML, Penín MA, Nemiña R, Cordido F, 2007 Metformin reduces thyrotropin levels in obese, diabetic women with primary hypothyroidism on thyroxine replacement therapy. Endocrine 32: 79-82.

11. Cappelli C, Rotondi M, Pirola I, et al, 2009 TSH-lowering effect of metformin in type 2 diabetic patients: differences between euthyroid, untreated hypothyroid, and euthyroid on L-T4 therapy patients. Diabetes Care 32: 1589-1590.

12. Cappelli C, Rotondi M, Pirola I, et al, 2012 Thyreotropin levels in diabetic patients on metformin treatment. Eur J Endocrinol 167: 261-265.

13. Díez JJ, Iglesias P, 2013 Relationship between serum thyrotropin concentrations and metformin therapy in euthyroid patients with type 2 diabetes. Clin Endocrinol (Oxf) 78: 505-511.

14. Morteza Taghavi S, Rokni H, Fatemi S, 2011 Metformin decreases thyrotropin in overweight women with polycystic ovarian syndrome and hypothyroidism. Diab Vasc Dis Res 8: 47-48.

15. Rotondi M, Cappelli C, Magri F, et al, 2011 Thyroidal effect of metformin treatment in patients with polycystic ovary syndrome. Clin Endocrinol (Oxf) 75: 378-381.

16. Alevizaki M, 2013 Metformin and the thyroid: some questions still remain. Clin Endocrinol (Oxf) 78: 503504.

17. Santos-Palacios S, Brugos-Larumbe A, Guillén-Grima F, Galofré JC, 2013 A cross-sectional study of the association between circulating TSH level and lipid profile in a large Spanish population. Clin Endocrinol (Oxf) 79: 874-881.

18. Rezzónico J, Rezzónico M, Pusiol E, Pitoia F, Niepomniszcze H, 2011 Metformin treatment for small benign thyroid nodules in patients with insulin resistance. Metab Syndr Relat Disord 9: 69-75.

19. Oleandri SE, Maccario M, Rossetto R, et al, 1999 Threemonth treatment with metformin or dexfenfluramine does not modify the effects of diet on anthropometric and endocrine-metabolic parameters in abdominal obesity. J Endocrinol Invest 22: 134-140.

20. Lupoli R, Di Minno A, Tortora A, Ambrosino P, Arianna Lupoli G, Di Minno MN, 2014 Effects of Treatment With Metformin on TSH Levels: A Meta-analysis of Literature Studies. J Clin Endocrinol Metab 99: E143-148.

21. Krysiak R, Okopien B, 2011 Thyrotropin-lowering effect of metformin in a patient with resistance to thyroid hormone. Clin Endocrinol (Oxf) 75: 404-406.

22. Bogachus LD, Turcotte LP, 2010 Genetic downregulation of AMPK-alpha isoforms uncovers the mechanism by which metformin decreases FA uptake and oxidation in skeletal muscle cells. Am J Physiol Cell Physiol 299: 1549-1561.

23. Duntas LH, Orgiazzi J, Brabant G, 2011 The interface between thyroid and diabetes mellitus. Clin Endocrinol (Oxf) 75: 1-9.

24. Fox CS, Pencina MJ, D’Agostino RB, et al, 2008 Relations of thyroid function to body weight: cross-sectional and longitudinal observations in a community-based sample. Arch Intern Med 168: 587-592. 